\title{
SIEGEL'S THEOREM FOR COMPLEX FUNCTION FIELDS
}

\author{
JOSÉ FELIPE VOLOCH
}

(Communicated by William Adams)

\begin{abstract}
We give a short proof of the finiteness of the set of integral points on an affine algebraic curve of genus at least one, defined over a function field of characteristic zero.
\end{abstract}

Siegel [Si] has shown that an affine algebraic curve of genus at least one defined over a number field has only finitely many integral points. Lang [L] has proven an analogous result for curves defined over a function field of characteristic zero not defined over the constant field. For curves of genus at least two, one even has the Mordell conjecture (proved by Faltings [F] in the number field case and by Manin [M] in the function field case) that there are only finitely many rational points.

For genus one, Manin [M] gave a proof of a strenghtening of Lang's result as a by-product of his work on the Mordell conjecture. Mason [Ms] then gave an effective proof by more elementary considerations. In this note, we give a short proof of Manin's (and hence Lang's) result for genus one. The proof can be adapted to higher genus as well (see the remark below).

Let $K$ be a function field with constant field of characteristic zero and $E / K$ an elliptic curve with nonconstant $j$-invariant. The reader may consult [S] for definitions and results about elliptic curves. In particular, we shall use the following results. The group $E(K)$ is a finitely generated abelian group by the Mordell-Weil theorem, and there is a height function $h: E(K) \rightarrow \mathbf{R}$ with the property that there are only finitely many points of bounded height ([L], Proposition 2). The height can be written as a sum of local heights $\sum \lambda_{v}(P)$, where $v$ ranges through the places of $K$. The local heights satisfy $\lambda_{v}(P)=$ $\max \{0, v(t(P))\}+\beta_{v}(P)$, where $\beta_{v}$ is bounded for all $v$ and is identically zero for all but finitely many $v$, and $t$ is a uniformizer at $0 \in E$. For example, $t=x / y$, where $x, y$ are coordinates of a Weierstrass equation for $E$.

Now, Lang's result for genus one can be reduced to the case of a Weierstrass equation ([S], Corollary IX.3.2.2) and in this case we argue as follows. Let $S$ be a finite set of places of $K$. Then $\sum_{v \notin S} \lambda_{v}(P)$ is bounded independently of $P$, if $P$ is $S$-integral and, since there are only finitely many points of bounded

Received by the editors October 5, 1992 and, in revised form, March 20, 1993.

1991 Mathematics Subject Classification. Primary 11G05, 11G30, 14G25.

Key words and phrases. Algebraic curves, function fields, integral points. 
height, if there are infinitely many $S$-integral points, then $\lambda_{v}$ is unbounded for some $v \in S$. It suffices thus to prove the following result:

Theorem (Manin). Let $K$ be a function field with constant field of characteristic zero and $E / K$ an elliptic curve with nonconstant $j$-invariant and $v$ a place of $K$. Then the local height function $\lambda_{v}$ is bounded on $E(K)$.

Proof. The points on $E\left(K_{v}\right)$ that reduce to $0 \bmod v$ form a subgroup $E_{1}\left(K_{v}\right)$ isomorphic to the group of points of a formal group. Choosing an uniformizer $t$, as above, on $E$ at 0 , then $E_{1}\left(K_{v}\right)=\left\{P \in E\left(K_{v}\right) \mid t(P) \in \mathscr{M}_{v}\right\}$, where $\mathscr{M}_{v}$ is the maximal ideal of the local ring at $v$. Moreover, $\lambda_{v}(P)$ differs from $v(t(P))$ by a bounded amount. Hence, it suffices to show that $v(t(P))$ is bounded above on $E(K)$. Suppose not and choose $P_{n}, n=1,2, \ldots$, in $E(K)$ such that $v\left(t\left(P_{n+1}\right)\right)>v\left(t\left(P_{n}\right)\right)>0$. We claim that $P_{1}, P_{2}, \ldots$ are linearly independent over $\mathbf{Z}$. Recall that $t$ induces a group isomorphism between $E_{r} / E_{r+1}$, where $E_{r}=E_{r}\left(K_{v}\right)=\left\{P \in E\left(K_{v}\right) \mid t(P) \in \mathscr{M}_{v}^{r}\right\}$, and $\mathscr{M}_{v}^{r} / \mathscr{M}_{v}^{r+1}$. If $n_{i} P_{i}=\sum_{j>i} n_{j} P_{j}, n_{i} \neq 0$, and $r=v\left(t\left(P_{i}\right)\right)$ then $n_{i} P_{i}$ is 0 in $E_{r} / E_{r+1}$, but $t\left(n_{i} P_{i}\right) \equiv n_{i} t\left(P_{i}\right) \not \equiv 0\left(\bmod \mathscr{M}_{v}^{r+1}\right)$, which proves the claim. On the other hand, the claim contradicts the Mordell-Weil theorem and this completes the proof.

Remark. On a curve of genus greater than one, if a sequence of points $P_{1}$, $P_{2}, \ldots$ approaches rapidly a point $P_{\infty}$, then a similar argument shows that the $P_{i}-P_{\infty}$ are linearly independent over $\mathrm{Z}$ in the Jacobian of the curve, and Lang's result follows from this. The author and A. Buium [BV] have recently proved a conjecture of Lang to the effect that an affine open subset of an abelian variety of any dimension over a function field of characteristic zero has finitely many integral points.

\section{REFERENCES}

[BV] A. Buium and J. F. Voloch, Integral points of abelian varieties over function fields of characteristic zero, Math. Ann. 297 (1993), 303-307.

[F] G. Faltings, Endlichkeitssätze für abelsche Varietäten über Zahlkörpen, Invent. Math. 73 (1983), 349-366.

[L] S. Lang, Integral points on curves, Inst. Hautes Études Sci. Publ. Math. 6 (1960), 27-43.

[M] Yu. I. Manin, Rational points on an algebraic curve over function fields, Izv. Acad. Nauk USSR (1963) and Letter to the editor, Math. USSR-Izv. 34 (1990), 465-466; English transl., Transl. Amer. Math. Soc. Ser. 2, vol. 50, Amer. Math. Soc., Providence, RI, 1966, pp. 189-234.

[Ms] R. C. Mason, Diophantine equations over function fields, London Math. Soc. Lecture Note Ser., vol. 96, Cambridge Univ. Press, Cambridge, 1984.

[S] J. H. Silverman, The arithmetic of elliptic curves, Graduate Texts in Math., vol. 106, Springer, New York, 1986.

[Si] C. L. Siegel, Einige Anwendungen diophantischer Approximationen, Abh. Preuss. Akad. Wiss. Phys. Math. Kl. 1 (1929), 41-69.

Department of Mathematics, University of Texas, Austin, Texas 78712-1082

E-mail address: voloch@math.utexas.edu 